\title{
A Comparative Evaluation of the Shear Bond Strength of Three Different Fifth Generation Dentin Bonding Agents: An in vitro Study
}

\author{
${ }^{1}$ Lata B Gangurde, ${ }^{2}$ Mansing G Pawar, ${ }^{3}$ Vanitha U Shenoy, ${ }^{4}$ Sumanthini V Margasahayam
}

\begin{abstract}
Purpose: To compare and evaluate the shear bond strength of three different fifth generation dentin bonding agents used with composite resin and to determine the mode of bond failure in each case.
\end{abstract}

Materials and methods: Forty-five freshly extracted human permanent molars were used and the specimens were divided into three test groups $(N=15)$ namely group $A=$ Excite, group $\mathrm{B}=$ Single Bond, group $\mathrm{C}=$ Prime and Bond NT. The occlusal surfaces of the specimens were ground to expose the superficial dentin and mounted in self cure acrylic resin. The surface was etched with $37 \%$ phosphoric acid gel and bonding agents were applied to etched surface. A composite button of $5.3 \times 3 \mathrm{~mm}$ ( $Z$ 100) was bonded to the test specimens and subjected to shear load using United Universal Testing Machine at the cross-head speed of $0.5 \mathrm{~mm} / \mathrm{min}$. The nature of bond failure was determined using a stereomicroscope at $\times 8$ magnification.

Results: Mean shear bond strength values in MPa of groups A, B, C were 21.483, 16.881 and 14.116 respectively. Group A showed $73.3 \%$ cohesive and $26.6 \%$ adhesive, Group B showed $66.6 \%$ adhesive, $20 \%$ cohesive and $13.33 \%$ mixed, group C showed $73.3 \%$ adhesive $6.6 \%$ cohesive and $20 \%$ mixed bond failures.

Conclusion: Excite dentin bonding agent exhibited highest shear bond strength values as compared to Single Bond and Prime and Bond NT, this difference was statistically significant. There was no statistically significant difference between the shear bond strength values of Single Bond and Prime and Bond NT. Maximum number of cohesive bond failure were observed in dentin with Excite. Single Bond and Prime and Bond NT exhibited maximum number of adhesive bond failure.

Keywords: Adhesion, Bonding agent, Moist dentin, Shear bond strength.

How to cite this article: Gangurde LB, Pawar MG, Shenoy VU, Margasahayam SV. A Comparative Evaluation of the Shear Bond Strength of Three Different Fifth Generation Dentin Bonding Agents: An in vitro Study. J Contemp Dent 2014;4(1):1-5.

\footnotetext{
${ }^{1}$ Reader, ${ }^{2}$ Dean, Professor and Head, ${ }^{3}$ Professor and Head ${ }^{4}$ Professor

1,3,4 Department of Conservative Dentistry, MGM Dental College and Hospital, Navi Mumbai, Maharashtra, India

${ }^{2}$ Department of Conservative Dentistry and Endodontics Government Dental College and Hospital, Mumbai Maharashtra, India

Corresponding Author: Lata B Gangurde, Reader Department of Conservative Dentistry, MGM Dental College and Hospital, Navi Mumbai, Maharashtra, India, Phone: 02227436604, e-mail: drlatagangurde@rediffmail.com
}

\section{Source of support: Nil}

Conflict of interest: None

\section{INTRODUCTION}

Dentistry emphasizes on preserving and maintaining the natural dentition. A fundamental objective of restorative procedures is to create adhesion between the mineralized tooth structure and the restorative material. One of the important requisites of a restorative material is that it should have capability to achieve a true permanent bond to the tooth structure and should possess mechanical properties such as strength. Bonding is the attachment of one substance to another. A bonding agent may be defined as a material that, when applied to the surface of substances, can join them together and resist separation. ${ }^{1}$

Buonocore, in 1955 introduced a technique of acid etching with phosphoric acid, which started a new era in the field of adhesive dentistry. ${ }^{2}$ Significant advances have occurred since then over last few decades. Recently, extensive research efforts have led to the development of bonding systems that not only bond the restorative material to the tooth structure but also to amalgam, composites, metals and ceramics. Dentin adhesives have progressed from the first generation, which had bond strengths of only 1-3 MPa to the present use of fifth, sixth and seventh generation bonding systems. ${ }^{3-5}$ The bonding systems most commonly used by dentists are fifth generation single component dentin bonding systems. This study was thought of to evaluate the shear bond strength of three such fifth generation dentin bonding agents.

The purpose of this in vitro study was to compare and evaluate the shear bond strength of three different fifth generation dentin bonding agents namely, Excite (Vivadent), Single Bond (3M), Prime and Bond NT (Dentsply) used in combination with composite resin and to determine the mode of bond failure in each case.

\section{MATERIALS AND METHODS}

Forty five freshly extracted non-carious human permanent molars extracted for periodontal reasons were collected for the study. Teeth were cleaned with ultrasonic scalers 
to remove all debris, stains and calculus and stored in distilled water at room temperature. The occlusal surfaces of the specimens were ground to expose the superficial dentin with the straight diamond point with water coolant. The specimens were then further wet ground with 180 grit silicon carbide carborundum paper, thus obtaining a flat ground occlusal surface perpendicular to the long axis of the tooth. Test specimens were then mounted in chemically cured acrylic resin using silastic mold. Care was taken to expose the occlusal dentin. The blocks were then immersed in water to dissipate the exothermic heat of polymerization. All the prepared test specimens were then further dry and wet ground with 320, 400, 600 grit silicon carbide carborundum paper respectively. All the prepared test specimens were then stored in distilled water. The specimens were then divided randomly into 3 groups (15 each), based on dentin bonding agents to be used in the study namely group $\mathrm{A}=$ Excite (Vivadent, Schaan /Liechtenstein), group B = Single Bond (3M Dental Products, USA), group $\mathrm{C}=$ Prime and Bond NT (Denstply, USA). The composition of dentin bonding agents and composite resin used in the study are as shown in Table 1. The surface of each specimen was etched with $37 \%$ phosphoric acid gel for 15 seconds, rinsed with water spray for 20 seconds and excess water was removed by blotting with a moist cotton pellet so that the tooth surface was left visibly moist. Bonding agents were then applied to the etched dentin surface according to the manufacturer's instructions and light cured for 20 seconds using the visible light curing unit. The light cured composite resin Z 100 (3M Dental Products, USA) was then placed on occlusal surface to prepare a composite button of $5.3 \times 3 \mathrm{~mm}$ with the help of silastic mold and was light cured for 40 seconds (Fig. 1). All the specimens were then stored in distilled water for 24 hours and subjected to shear load using United Universal Testing Machine (United Calibration Corp, 5802, Engineer Dr, Huntington Beach CA, Model No. STM. 20. PC, Serial No. 1294575) at the cross-head speed of $0.5 \mathrm{~mm} / \mathrm{min}$. The force was applied till the bond failure occurred. The maximum load to debond the specimen was recorded in Newtons. Shear bond strength was calculated in megapascal units $(\mathrm{MPa})$ by the ratio of maximum load in
Newtons to the cross sectional area of the bonded interface in millimeters. After the shear bond testing, the debonded specimens of all the groups were examined under a stereomicroscope at $\times 8$ magnification to determine the nature of bond failure. Observation were tabulated and statistical analysis was done using 't' test and p-value $<0.001$.

\section{RESULTS}

Group A containing Excite dentin bonding agent exhibited shear bond strength values in the range of 15.94624.367 MPa with a mean shear bond strength value of 21.4831 MPa. Group B containing Single Bond dentin bonding agent exhibited shear bond strength values in the range of 12.794-19.999 $\mathrm{MPa}$ with a mean shear bond strength value of 16.8814 MPa. Group C containing Prime and Bond NT dentin bonding agent exhibited shear bond strength values in the range of 9.380-17.726 MPa with a mean shear bond strength value of $14.116 \mathrm{MPa}$. The mean shear bond strength values in $\mathrm{MPa}$ is as shown in Graph 1. The mean and standard deviation value for shear bond strength in MPa of three groups are given in Table 2.

Based on statistical analysis, group A showed statistically significant difference in shear bond strength when compared with groups B and C. There was no statistically significant difference between the shear bond strength of groups B and $\mathrm{C}$. The comparison between three groups is given in Table 3.

In group A, $73.3 \%$ of specimens showed cohesive bond failure (Fig. 2) and $26.6 \%$ of them showed adhesive bond failure. Group B, specimens showed $66.6 \%$ adhesive failure (Fig. 3) whereas 20\% showed cohesive and $13.33 \%$ showed mixed failure (Fig. 4). Group C showed 73.3\% adhesive failures whereas cohesive failures and mixed failures were 6.6 and $20 \%$ respectively. The mode of bond failure of each group is presented in Table 4.

\section{DISCUSSION}

The foundation of adhesive dentistry was laid in 1955 when Buonocore proposed that the acids could be used to alter the

Table 1: Composition of bonding agent and composite used in this study

\begin{tabular}{|c|c|c|c|}
\hline Excite & Single bond & Prime and Bond NT & Z 100 Composite resin \\
\hline $\begin{array}{l}\text { Phosphoric } \\
\text { acid acrylate, } \\
\text { dimethacrylates, } \\
\text { HEMA, Bis-GMA, } \\
\text { highly dispersed } \\
\text { silicon dioxide, } \\
\text { ethanol, catalysts, } \\
\text { stabilizers }\end{array}$ & $\begin{array}{l}\text { Water, ethanol, } \\
\text { 2-hydroxyethyl methacrylate } \\
\text { (HEMA), bis-phenol A } \\
\text { diglycidylmethacrylate } \\
\text { (Bis-GMA), photoinitiator, } \\
\text { methacrylate functional } \\
\text { copolymer of polyacrylic and } \\
\text { polyitaconic acid }\end{array}$ & $\begin{array}{l}\text { - } \text { PENTA } \\
\text { - } \text { UDMA } \\
\text { - } \text { Butylated hydroxytoluene } \\
\text { - } \text { 4-ethyl dimethacrylate amino benzoate } \\
\text { - } \text { Cetylamine hydroxyfluoride } \\
\text { - } \text { Silica nano filler } \\
\text { - Camphoroquinone } \\
\text { - Solvent } \\
\text { - Acetone }\end{array}$ & $\begin{array}{l}\text { The matrix of this composite is } \\
\text { a resin consisting of Bis-GMA } \\
\text { (bisphenol A diglycidyl ether } \\
\text { dimethacrylate) and TEGDMA } \\
\text { [tri (ethylene glycol) dimethacrylate]. } \\
\text { This light-cured resin is filled with } \\
66 \% \text { (volume) silica/zirconia. The } \\
\text { filler average particle size is } 0.6 \mu \mathrm{m}\end{array}$ \\
\hline
\end{tabular}


surface of enamel, 'render it more receptive to adhesion'2 He found that acrylic resin could be bonded to human enamel that was conditioned with $85 \%$ phosphoric acid for 30 seconds. Significant advances have occurred since then over past few decades. The bonding of Bis-GMA to etched enamel introduced esthetic restorations without the need for mechanical retention within cavity preparation. Early attempts to bond dentin resulted in poor bond strengths. ${ }^{6}$ The improvements and current development in bonding agents resulted in different generations of dentin bonding systems. ${ }^{7}$ Several studies clearly showed that current generation of dentin bonding systems provides higher bond strengths to dentin. ${ }^{3-5}$

The fifth generation of bonding system was developed to make the use of adhesive material more reliable for practitioners. It consists of two different types of adhesive materials, 'one bottle systems and self-etching primer bonding system'. In the present study, three fifth generation one bottle dentin bonding agents were used. These bonding agents have the combination of primer and adhesive into one solution which is to be applied after etching enamel

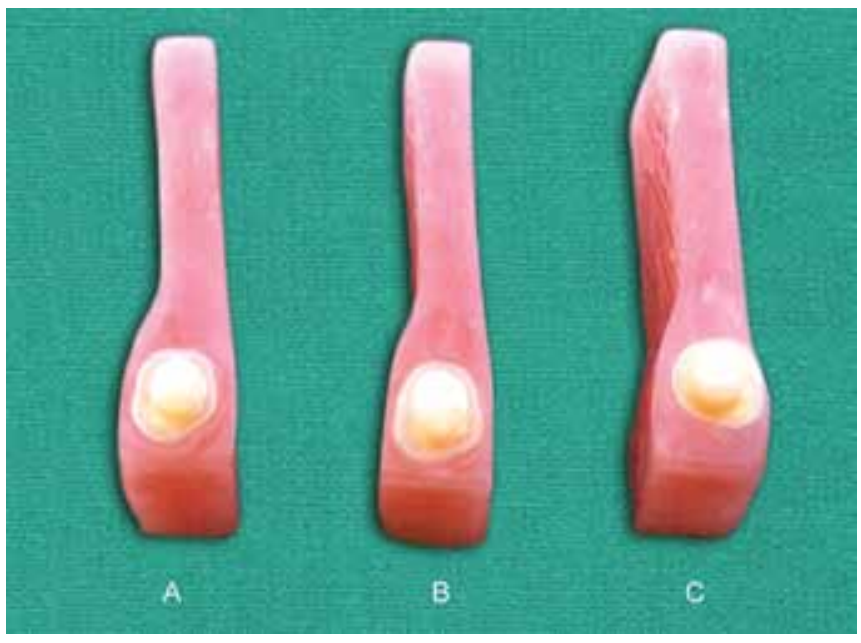

Fig. 1: Experimental groups of prepared specimens

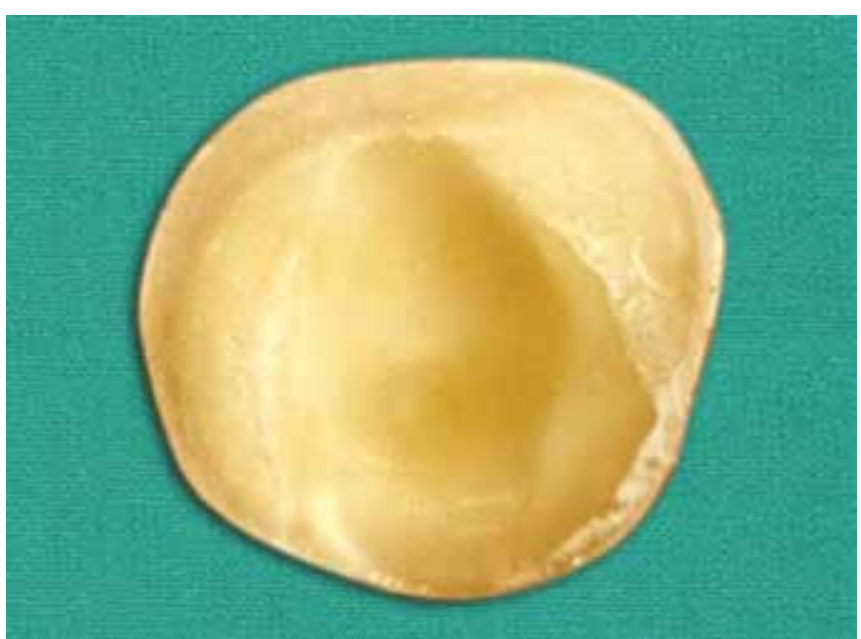

Fig. 2: Specimen showing cohesive failure and dentin simultaneously (total etch). These bonding agents create mechanical interlocking with etched dentin by means of resin tags, adhesive lateral branches and hybrid layer formation and show high bond strength values both to etched enamel and dentin. These bonding agents simplify clinical procedure by reducing the bonding step and thus

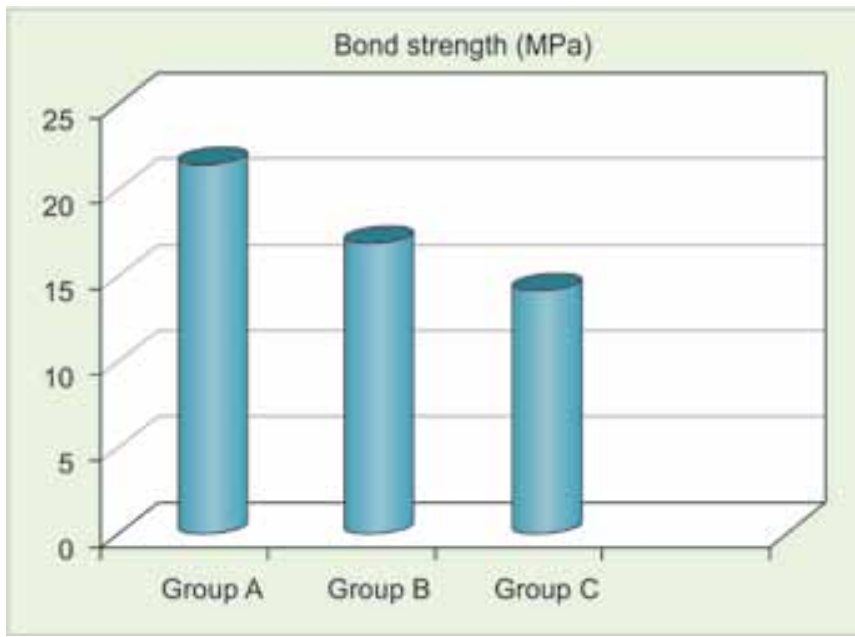

Graph 1: Mean shear bond strength in MPa (group A: Excite, group B: Single Bond, group C: Prime and Bond NT)

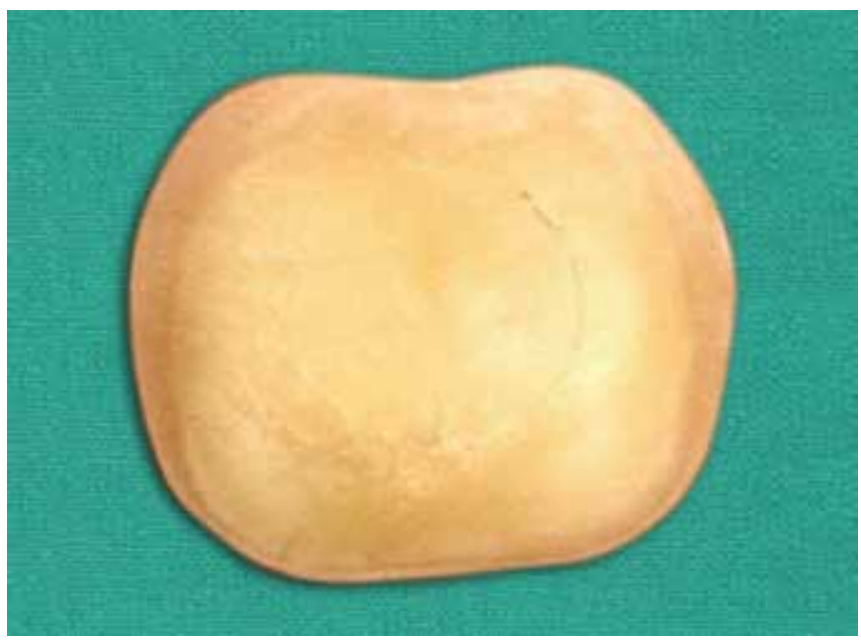

Fig. 3: Specimen showing adhesive failure

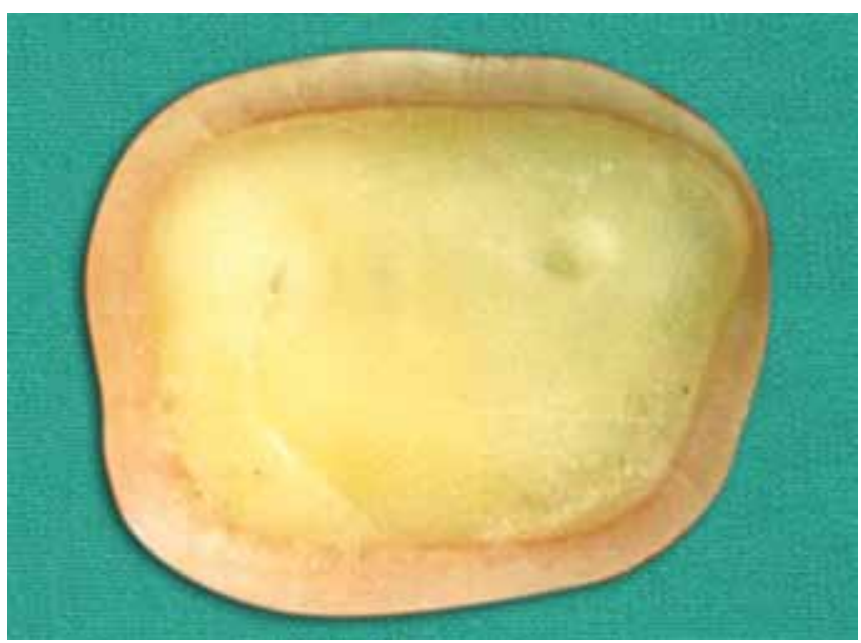

Fig. 4: Specimen showing mixed failure 
working time and also help to prevent collagen collapse of demineralized dentin.

In the present study, group A showed highest bond strength with a mean of $21.483 \mathrm{MPa}$, when compared with specimens of groups $\mathrm{B}$ and $\mathrm{C}$ showing mean shear bond strength values of $16.881 \mathrm{MPa}$ and 14.116 MPa respectively. Based on statistical analysis, group A showed statistically significant difference in shear bond strength when compared with groups $\mathrm{B}$ and $\mathrm{C}$. There was no statistically significant difference between the shear bond strengths of groups B and $\mathrm{C}$. The bonding agent, Excite contains hydroxyethylmetha acrylates (HEMA), dimethacrylates, phosphoric acid acrylates, highly dispersed silicone dioxide, initiators and stabilizers in an alcohol solution which reacts quickly and ensures long-term stability. Several studies reported that 2-HEMA monomer when applied to conditioned dentinal surface enhances the bond strength which is suggestive of highest bond strength of this group. ${ }^{8-11}$ Excite being based on an alcohol solvent, its application is more technique tolerant as it is less volatile than acetone and is not as greatly affected by the degree of dentin moisture. Acetone may lead to excessive dehydration of dentin and it evaporates quickly. Excite being acetone free solvent represents a good compromise between water and highly volatile solvent, such as acetone.

Single Bond contains a solution of water, ethanol, HEMA, Bis-GMA, dimethacrylates, photoinitiators, a methacrylate functional copolymer of polyacrylic and polyitaconic acid. The affinity of HEMA for dentin seems to be enhanced when it is combined with water displacing solvents like ethanol which can displace water from the dentin surface and permit infiltration of monomer through nanospaces of a

Table 2: Mean shear bond strength (MPa)

\begin{tabular}{llll}
\hline Group & \multicolumn{3}{c}{ Bond strength } \\
\cline { 2 - 4 } & Mean & SD & Range $(\min \rightarrow$ max $)$ \\
\hline $\mathrm{A}(\mathrm{N}=15)$ & 21.4831 & \pm 2.2762 & $15.946 \rightarrow 24.367$ \\
$\mathrm{~B}(\mathrm{~N}=15)$ & 16.8814 & \pm 2.6422 & $12.794 \rightarrow 19.999$ \\
$\mathrm{C}(\mathrm{N}=15)$ & 14.1116 & \pm 2.9398 & $9.380 \rightarrow 17.726$ \\
\hline
\end{tabular}

dense collagen web hence enhancing bond strength. ${ }^{16}$ It has been suggested that the water in the composition of some adhesives would be able to reopen the collapsed network of collagen fibers on dry spots inadvertently left on the surface and prevent the formation of ghost hybrid layers. ${ }^{12,13}$ The results of the shear bond strength obtained in the present study are in accordance with other studies reported in the literature. ${ }^{14-16}$

Prime and Bond NT is a acetone based adhesive and contains Di and Trimethacrylic resin, PENTA (dipentacrythritolpenta acrylate monophosphate), Nanofillers, Amorphous silicon dioxide photoinitiators, stabilizers, cetylamine hydrofluoride. It requires a moist dentin surface to produce adequate bonding. It acts as a water chaser and helps diffusion of primer into dentin and substrate. ${ }^{16}$ It being sensitive to amount of water on the dentin surface, even a small amount of drying may have a significant role in reducing the bond strength. In shear bond strength studies of different versions of Prime and Bond NT such as Prime and Bond 2.0, ${ }^{17}$ which has similar composition of Prime and Bond NT except for the addition of fluoride, Prime and Bond $2.1^{15}$ was comparable to the one used in the current study. However Jeorge P reported a lower shear bond strength of Prime and Bond 2.1 in their study. ${ }^{14}$ Studies conducted by Vijay M, Mano CA showed the shear bond strength values for Single Bond and Prime and Bond NT are comparable with the results of present study. ${ }^{18}$

Following the shear bond strength testing, the debonded specimens of groups A, B, C were examined at $8 \times$ magnification using a stereomicroscope to determine the mode of bond failure between the adhesive material and dentin. Bond failure were recorded as adhesive (those occurred between dentin bonding agent and dentin), cohesive (those which occurred with in either the dentin, dentin bonding agent or composite resin) or mixed those which were a combination of adhesive and cohesive). The comparative evaluation of cohesive $v s$ adhesive failure between the resin and dentin demonstrates excellent adhesive characteristics of newer dentin bonding systems.

Table 3: Comparison of shear bond strength between groups

\begin{tabular}{lllll}
\hline Groups compared & Mean difference & SE & 't'value & Significance \\
\hline A vs B & 4.6017 & \pm 0.9320 & 4.9374 & $p<0.001$ \\
A vs C & 7.3715 & \pm 0.9936 & 7.4189 & $p<0.001$ \\
B vs C & 2.7698 & \pm 1.0563 & 2.26221 & $p<0.001$ \\
\hline
\end{tabular}

By ' $t$ ' test $p<0.001$

Table 4: Failure modes of each group

\begin{tabular}{|c|c|c|c|c|c|c|c|c|}
\hline \multicolumn{3}{|c|}{ Group A-Excite } & \multicolumn{3}{|c|}{ Group B-Single Bond } & \multicolumn{3}{|c|}{ Group C_-Prime and Bond NT } \\
\hline$A$ & $C$ & $M$ & $A$ & $C$ & $M$ & $A$ & $C$ & $M$ \\
\hline 4 & 11 & 0 & 10 & 3 & 2 & 11 & 1 & 3 \\
\hline $26.6 \%$ & $73.3 \%$ & - & $66.66 \%$ & $20 \%$ & $13.33 \%$ & $73.3 \%$ & $6.6 \%$ & $20 \%$ \\
\hline
\end{tabular}

A: Adhesive; C: Cohesive in dentin or composite; M: Mixed adhesive/cohesive 


\section{CONCLUSION}

The present study was undertaken to comparatively evaluate the shear bond strength of three fifth generation dentin bonding agents namely Excite (Vivadent), Single Bond (3M), Prime and Bond NT (Denstply).

Based on the results following conclusions can be drawn:

1. Excite dentin bonding agent exhibited highest shear bond strength values in comparison to the shear bond strength values of Single Bond and Prime and Bond NT dentin bonding agents and this difference was statistically significant $(\mathrm{p}<0.001)$.

2. When Single Bond and Prime and Bond NT were compared with respect to their shear bond strength, no statistically significant difference $(\mathrm{p}<0.001)$ was observed.

3. Maximum number of cohesive bond failures were observed in dentin with excite dentin bonding agent, whereas, Single Bond and Prime and Bond NT exhibited maximum number of adhesive bond failure.

\section{FUTURE SCOPE}

The use of adhesives in dentistry has been progressing at a rapid rate resulting in the rapid development of many new products. Development of adhesive dentistry is now focused on gaining a better understanding factors affecting adhesion in oral environment and to improve the clinical longevity of restorative materials. Further research is required to evaluate the long-term in vivo performance of current generation of dentin bonding agents.

\section{REFERENCES}

1. Kinloch AJ, Hall CP. A review of adhesion and adhesives: science and technology. London and New York 1987, 441. The Journal of Adhesion 1989;28.

2. Buonocore MG. A simple method of increasing the adhesion of acrylic filling materials to enamel surfaces. J Dent Res 1995;34:849-853.
3. Davidson CL. An investigation into the quality of dentin bonding systems for accomplishing a durable bond. Journal of Oral Rehab 1993;20:291-300.

4. Tyas MJ. Clinical evaluation of five adhesive systems. Am J Dent 1994; 7:77-80.

5. Van Meerbeek B. Clinical status of ten dentin adhesive systems. J Dent Res 1994;73:1690-1702.

6. Bowen RL. Dental filling materials comprising vinyl-silane treated fused silica and a binder consisting of the reaction product of bisphenol and glycidylmethacrylate. US Patent 1962;3:066,112.

7. Cerard K, Ferrari M. The science of bonding: from first to sixth generation. J Am Dent Assoc 2000;131:20-25.

8. Nakabayashi N. Effect of HEMA on bonding to dentin. Dent Mater 1992;8:125-130.

9. Gerbeno C, Nakabayashi N. Effect of HEMA treatment on bonding to EDTA pretreated dentin. J Dent Res 1992;71:615 (abstract 798).

10. Erikson RL. Mechanism and clinical implication of bond formation for two dentin bonding agents. Am J Dent 1989;2: $117-123$

11. Munksgarrd EC, Asmussen E. Bond strength between dentin and restorative resins mediated by mixtures of HEMA and Glutaraldehyde. J Dent Res 1984;63:1087-1089.

12. Van Meerbeek B. A TEM study of two water based adhesive systems bonded to dry and wet dentin. J Dent Res 1998;77: 50-59.

13. Tay FR. Micromorphological spectrum from overdrying to overwetting acid conditioned dentin in water-free, acetonebased, single-bottle primer/ adhesives. Dent Mater 1996;12: 236-244.

14. Perdigao J. In vitro interfacial relationship between human dentin and one-bottle dental adhesives. Dent Mater 1997;13: 218-227.

15. Ali Al Ehaideb, Mohammed H. Shear bond strength of one bottle dentin adhesives. J Prosthet Dent 2000;84:408-412.

16. Jonathan R, Lakshminarayanan L. Comparison of shear bond strength of fifth generation dentin bonding agents: in vitro study. J Cons Dent 2001;4:122-129.

17. Fritz UB, Finger WJ. Resin modified glass ionomer cements: bonding to enamel and dentin. Dent Mater 1996;12:161-166.

18. Vijay M, Angelo MC, Mariamma KC, Jayakumar K, Sarath BK. Comparative evaluation of the effect of $15 \%$ carbamide peroxide bleaching on shear bond strength using three different adhesive systems: an in vitro study. IJAPBS 2013;2:18-23. 